\title{
Anesthetic Management for a Patient with Congenital Insensitivity to Pain with Anhidrosis (CIPA) - A case report -
}

Department of Anesthesiology and Pain Medicine, Chonnam National University Medical School, Gwangju, Korea

Seok Jai Kim, M.D., Kyung Yeon Yoo, M.D., Myung-Woo Kang, M.D., Jeen Wook Hong, M.D., Hyung-Gon Lee, M.D., Dong-Jin Shin, M.D., and Woong-Mo Kim, M.D.

Congenital insensitivity to pain with anhidrosis (CIPA) is a rare, autosomal-recessive disorder characterized by the clinical triad of indifference of pain, anhidrosis and heat intolerance. Because of their lack of autonomic response to noxious stimuli, the determination of adequate depth of anesthesia in the CIPA patient undergoing surgery is a major challenge. We experienced a patient with CIPA who had minor procedures three times under the general anesthesia, in which bispectral index (BIS) was maintained at $40-50$ by adjusting sevoflurane concentrations with $50 \%$ nitrous oxide. The low end-tidal sevoflurane concentrations $(<1.2$ vol\%) were required to keep the target BIS while vital signs remained stable throughout the surgery in each operation. BIS monitor may be a valuable tool to guide the depth of anesthesia in patients with CIPA. (Korean J Anesthesiol 2008; 54: S 47 50)

Key Words: bispectral index, congenital insensitivity to pain and anhidrosis, volatile anesthetics.

Congenital insensitivity to pain and anhidrosis (CIPA) is a rare, autosomal-recessive disorder and classified as hereditary sensory and autonomic neuropathy type IV. ${ }^{1)}$ Patients with CIPA are characterized by abnormal responses to painful stimuli, recurrent episodic fevers or heat intolerance, anhidrosis, and mental retardation. Insensitivity to pain may lead to bone fracture, multiple scars, osteomyelitis and joint deformities and patients may require surgery such as osteotomy and amputation. Although they do not feel pain, patients with CIPA need anesthesia to undergo surgical procedures because they often display tactile hyperesthesia, which is thought to compensate for alganesthesia. Moreover, anxiety can induce stress that may cause perioperative instability. ${ }^{2,3)}$ However, the adequate level of anesthesia needed for patients with CIPA has yet to be determined. We report a case of a 6-year-old boy with CIPA who underwent minor procedures three times where a bispectral index score (BIS) monitor was used to guide the anesthetic

Received : October 11, 2007

Corresponding to : Kyung Yeon Yoo, Department of Anesthesiology and Pain Medicine, Chonnam National University Medical School, 8, Hak-dong, Dong-gu, Gwangju 501-757, Korea. Tel: 82-62-220-6893, Fax: 82-62-232-6294, E-mail: kyyoo@ chonnam.ac.kr depth.

\section{CASE REPORT}

A 6-year-old, $14 \mathrm{~kg}, 106 \mathrm{~cm}$ boy was scheduled for skin graft at left knee as the first operation and then, two times of curettage and debridement for wound infection at right knee. He had a certain degree of mental retardation but had no familial history of CIPA. He experienced left tibiofibular fracture at the age of 2 and left hip dislocation and right calcaneal fracture next year. In addition, he had hyperkeratosis at his palms and soles, extensive interdigital skin fissures, and poorly healed minor skin wounds (Fig. 1, 2). He had no abnormal findings in hematological, chemistrical and electromyographical examinations. The diagnosis of CIPA was confirmed by sural nerve biopsy 2 years before (Fig. 3).

$\mathrm{He}$ was premedicated with diazepam $4 \mathrm{mg}$ orally before induction of anesthesia in each operation. Upon arrival in the operating room, a 22-gauge catheter was inserted into a radial artery to measure blood pressure and to take blood samples. BIS monitor strip (BIS Sensor-Aspect Medical Systems, Newton, MA) was placed on the forehead and BIS values were recorded with a BIS monitor (2000A, Aspect Medical 
systems Inc., USA). After obtaining baseline values, anesthesia was induced with $5 \mathrm{mg} / \mathrm{kg}$ IV thiopental, followed by 0.7 $\mathrm{mg} / \mathrm{kg}$ IV rocuronium. Intubation was performed when neuromuscular block was achieved, and anesthesia was maintained with sevoflurane and 50\% nitrous oxide in oxygen. The end-tidal sevoflurane concentrations were adjusted to maintain BIS at $40-50$ throughout the surgery and they were less than 1.2 vol\% in every operation. The patient was mechanically ventilated using a ventilator to maintain an end-tidal carbon dioxide tension between 35 and $40 \mathrm{mmHg}$. Neuromuscular blockade was carefully controlled by monitoring train-of-four, and additional boluses of rocuronium were administered to maintain one response during the surgical procedure. The operating room temperature was maintained at $21^{\circ} \mathrm{C}$. Routine monitoring included invasive measurement of systemic arterial pressure, heart rate and rhythm by 5-lead electrocardiogram, and oxygen saturation by pulse oximetry. Throughout the experiment, the end-tidal concentrations of carbon dioxide,
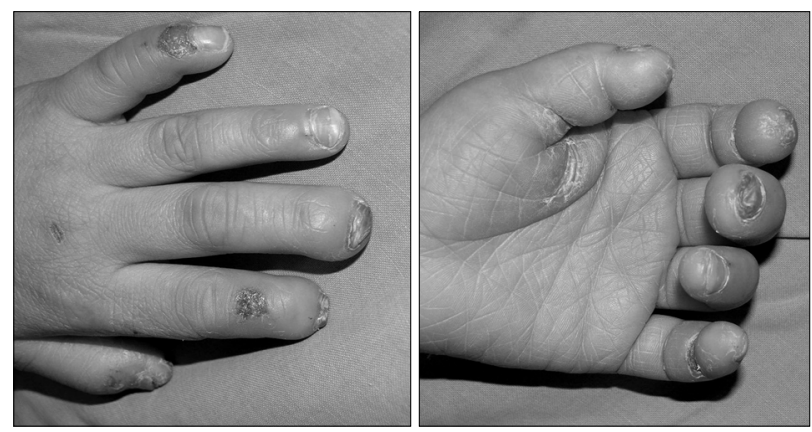

Fig. 1. Photograph of left hand showing hyperkeratosis and ulceration with extensive interdigit skin fissuring. sevoflurane and nitrous oxide were measured using a gas analyzer (Capnomac Ultima; Datax, Helsinki, Finland). Body temperature was monitored with transesophageal and tympanic membrane thermometer throughout the operation and remained within normal limits $\left(36.2-36.5^{\circ} \mathrm{C}\right)$. Pyridostigmine $2 \mathrm{mg}$ and glycopyrrolate $0.15 \mathrm{mg}$ were given for reversal of neuromuscular blockade at the end of procedure. The patient was extubated and transferred to the postanesthesia care unit. There was no emergence excitement, fever or pain in the postoperative recovery period.

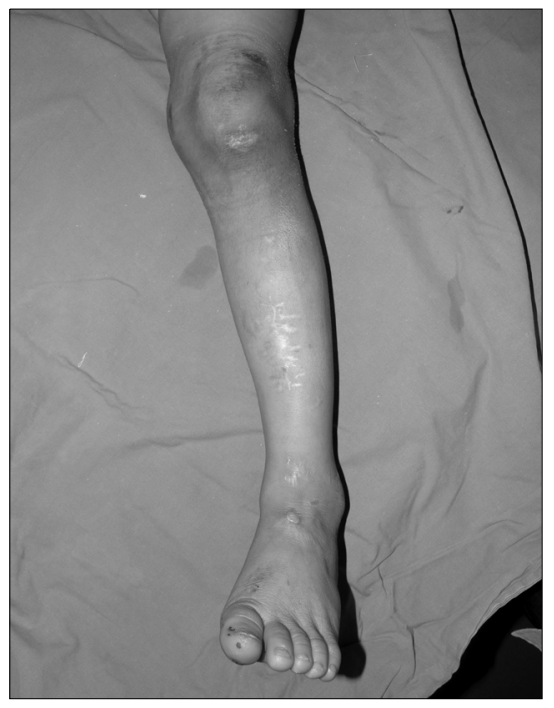

Fig. 2. Photograph of left leg showing hyperkeratosis and ulceration with poor healing skin wounds.
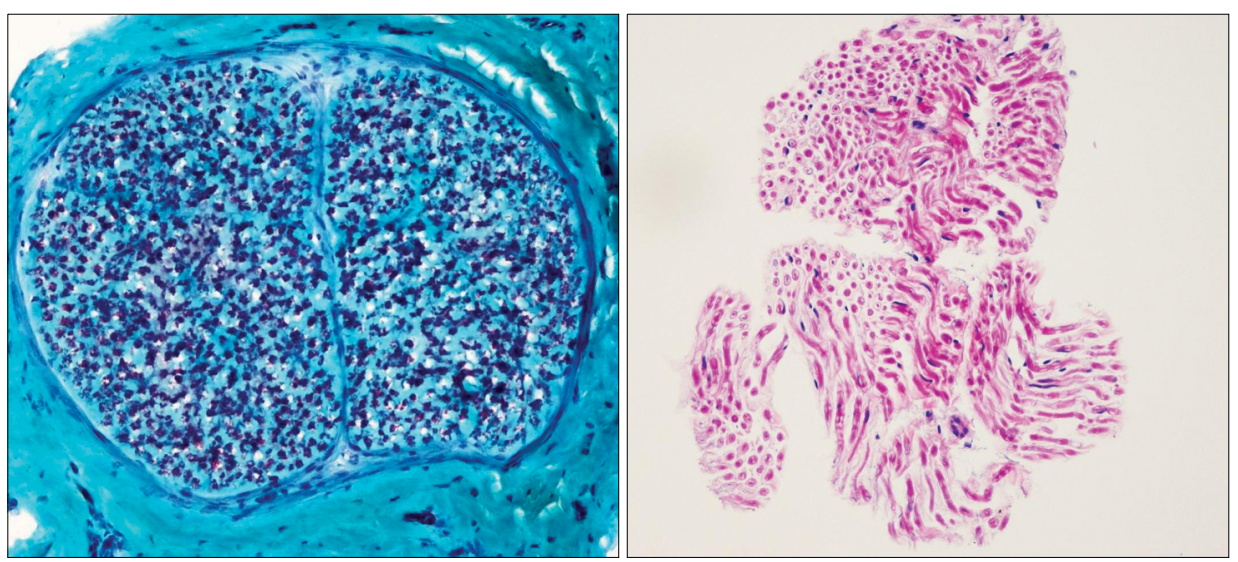

S 48
Fig. 3. Microscopic findings showing abundant large myelinated fibers and few number of small myelinated and unmyelinated fibers. 


\section{DISCUSSION}

CIPA, first reported by Nishida in $1951{ }^{4}$ ) is a hereditary disease caused by mutations in the neurotrophic tyrosine receptor kinase 1 gene (NTRK1 gene, previously known as trkA gene). Since neurotrophic tyrosine receptor is a receptor for nerve growth factor (NGF), ${ }^{5)}$ there is a failure of differentiation and migration of neural crest cells resulting in the complete absence of small myelinated and unmyelinated nerve fibers in patients with CIPA. ${ }^{6}$ As a result, there are defects in pain sensation and impairment of peripheral sympathetic nerves. A defect in pain sensation causes analgesia and the lack of peripheral sympathetic innervation of the sweat glands cause anhidrosis and, consequently, body temperature regulation is disturbed. Our patient had experienced multiple trauma due to insensitivity to pain and developed chronic orthopedic problems requiring frequent surgery from lack of appropriate nociceptive feedback. Main goals of anesthetic management in patients with CIPA includes the prevention of psychological stress by minimizing apprehension and anxiety, maintenance of normal body temperature in the perioperative period, and adjustment of the anesthetic depth to prevent intraoperative awareness while avoiding high concentration that may cause hypotension, bradycardia or cardiac arrest. ${ }^{7,8)}$

It has been recommended to use benzodiazepines to relieve patients from preoperative anxiety. The premedication helps to relieve patients from anxiety and also reduce the risk of fever caused by excitement. In our case, the patient was premedicated with diazepam $4 \mathrm{mg}$ orally and when he arrived at operating room, he did not agitate himself. Because of the presence of anhidrosis, it has been recommended to avoid anticholinergics in patients with CIPA. ${ }^{9)}$ However, our patient received this medication in the operating room to reverse muscle relaxation without any adverse effects, being consistent with a previous study. ${ }^{10}$ ) In normal subjects, anticholinergics inhibit the activity of sweat glands and sweating may be sufficiently depressed to raise the body temperature. ${ }^{10)}$ In contrast, CIPA patients already do not have the ability to sweat. Therefore, it is unlikely the use of anticholinergics would affect the body temperature in CIPA patients.

Because CIPA patients are not capable of sweating, controlling body temperature is another crucial perioperative task. They rely on a strict management of environmental temperature to maintain normal body temperature and are at risk of both hyperthermia and hypothermia. Therefore, anesthesiologists should pay attention to temperature in the operating and recovery room. We used a heating/cooling blanket on our patient during the surgical procedure and a convective warming device (Thermacare convective warming unit TC 3000, Gaymar Industries Incorperated, Orchard Park, NY) in the recovery room. The patient's body temperature should be monitored very closely. Throughout the perioperative period, we monitored esophageal and tympanic membrane temperatures and the body temperature was remained within normal limits.

It has been proposed that patients with CIPA may require less anesthesia because they do not feel pain. ${ }^{11)}$ However, Okuda et al. ${ }^{12)}$ reported that the anesthetic requirements differed according to the type of surgeries. In their report, the required concentration of anesthetic was over 1.0 MAC for orthopedic surgery, and less than 1.0 MAC for dental surgery. They speculated that the different anesthetic concentrations is related to the different intensity of nociceptive stimulus of each procedure. Moreover, some patients did complain of pain in the postoperative period. Although pain sensation and temperature sense is severely impaired in patients with CIPA, they may still feel noxious surgical stimuli. Therefore, it appears desirable that the patients should receive the different depth of anesthesia according to surgical stimulus and the individual need. Nevertheless, a deep level of anesthesia should be avoided because of the possible occurrence of severe hypotension or cardiac arrest. Indeed, Rozentsveig et al. ${ }^{13)}$ reported a case of death of a toddler who had intraoperative cardiac arrest under anesthesia of relatively high concentration of halothane $(>2 \mathrm{MAC})$ and $66 \%$ nitrous oxide for minor orthopedic procedure. Lee et al. ${ }^{14)}$ introduced total intravenous anesthesia using propofol and remifentanil for a patient with CIPA, and reported that the anesthetic technique had advantages such as rapid emergence, attenuation of postoperative anxiety, nausea and vomiting. Jang et al. ${ }^{15}$ introduced volatile induction and maintenance of anesthesia using sevoflurane and nitrous oxide without neuromuscular blocker, and reported the technique had no necessity to reverse muscle relaxation with anticholinergics which may inhibit thermoregulation. However, both techniques ${ }^{14,15)}$ can not avoid a deep level of anesthesia, which may be dangerous for CIPA patients.

An inhaled anesthetic requirement is generally quantified by either a lack of response in terms of movement or by a lack of hemodynamic responses. However, in patients with CIPA, both of these responses are altered, i.e., they do not increase 
heart rate and blood pressure in response to painful stimuli because they have abnormal autonomic function, ${ }^{2)}$ and they do not move even in the absence of adequate anesthesia because they generally receive neuromuscular blocker to prevent movement under the light anesthesia. BIS value less than 50 indicates an adequate depth of hypnosis to prevent recall for a variety of clinically-used anesthetic agents in adults. ${ }^{16)}$ Several studies have shown that BIS is adequately calibrated for use in children older than 1 year. ${ }^{17,18)}$ Therefore, we used the amount of sevoflurane required to maintain a BIS of 40-50 irrespective of the adequateness of anesthesia during the surgery. Throughout the procedures, the vital signs were stable in every operation, as has been demonstrated in a previous study. These results suggest that BIS is a reliable monitor for the titration of anesthetics to ensure unconsciousness in pediatric CIPA patients while avoiding excessive concentrations.

In conclusion, anesthesia is necessary to provide unconsciousness and to prevent stress responses in patients with CIPA. BIS monitor can be a useful tool to guide the adequate depth of anesthesia for these patients.

\section{REFERENCES}

1. Nagasako EM, Oaklander AL, Dworkin RH: Congenital insensitivity to pain: an update. Pain 2003; 101: 213-9.

2. Rosemberg S, Marie SK, Kliemann S: Congenital insensitivity to pain with anhidrosis (hereditary sensory and autonomic neuropathy type IV). Pediatr Neurol 1994; 11: 50-6.

3. Holm-Knudsen RJ, Carlin JB, McKenzie IM: Distress at induction of anaesthesia in children. A survey of incidence, associated factors and recovery characteristics. Pediatr Anaesth 1998; 8: 383-92.

4. Nishida G: Congenital anhidrosis. Saishin Igaku (Japan) 1951; 6: $1100-4$.

5. Indo Y, Tsuruta M, Hayashida Y, Karim MA, Ohta K, Kawano T, et al: Mutations in the TRKA/NGF receptor gene in patients with congenital insensitivity to pain with anhidrosis. Nat Genet 1996; 13: 485-8.
6. Bonkowsky JL, Johnson J, Carey JC, Smith AG, Swoboda KJ: An infant with primary tooth loss and palmar hyperkeratosis: a novel mutation in the NTRK1 gene causing congenital insensitivity to pain with anhidrosis. Pediatrics 2003; 112: 237-41.

7. Brandes IF, Stuth EA: Use of BIS monitor in a child with congenital insensitivity to pain with anhidrosis. Pediatr Anaesth 2006; 16: 466-70.

8. Tomioka K, Awaya Y, Nihei K, Sekiyama H, Sawamura S, Hanaoka K: Anesthesia for patients with congenital insensitivity to pain and anhidrosis: a questionnaire study in Japan. Anesth Analg 2002; 94: 271-4.

9. Pinsky L, DiGeorge AM: Congenital familial sensory neuropathy with anhidrosis. J Pediatr 1966; 68: 1-13.

10. Hardman JG, Gilman AG, Limbird LE: Goodman \& Gilman's the pharmacological basis of therapeutics. 9th ed. New York, McGraw-Hill. 1996, p 153.

11. Mitaka C, Tsunoda Y, Hikawa Y, Sakahira K, Matsumoto I: Anesthetic management of congenital insensitivity to pain with anhidrosis. Anesthesiology 1985; 63: 328-9.

12. Okuda K, Arai T, Miwa T, Hiroki K: Anaesthetic management of children with congenital insensitivity to pain with anhidrosis. Paediatr Anaesth 2000; 10: 545-8.

13. Rozentsveig V, Katz A, Weksler N, Schwartz A, Schilly M, Klein $\mathrm{M}$, et al: The anaesthetic management of patients with congenital insensitivity to pain with anhidrosis. Pediatr Anaesth 2004; 14: 344-8.

14. Lee SJ, Kim YK, Seong KW, Kim SW: Total intravenous anesthetic management of a child with congenital insensitivity to pain with anhidrosis. Korean J Anesthesiol 2007; 52: 367-70.

15. Jang YH, Park JH, Song KS: Anesthetic management in a patient with congenital insensitivity to pain with anhidrosis. Korean $\mathrm{J}$ Anesthesiol 2004; 47: 281-3.

16. Katoh T, Suzuki A, Ikeda K: Electroencephalographic derivatives as a tool for predicting the depth of sedation and anesthesia induced by sevoflurane. Anesthesiology 1998; 88: 642-50.

17. Denman WT, Swanson EL, Rosow D, Ezbicki K, Connors PD, Rosow CE: Pediatric evaluation of the bispectral index (BIS) monitor and correlation of BIS with end-tidal sevoflurane concentration in infants and children. Anesth Analg 2000; 90: 872-7.

18. Whyte SD, Booker PD: Bispectral index during isoflurane anesthesia in pediatric patients. Anesth Analg 2004; 98: 1644-9. 